\title{
Systematic micro-array based identification of placental mRNA in maternal plasma: towards non-invasive prenatal gene expression profiling
}

\author{
N B Y Tsui, S S C Chim, R W K Chiu, T K Lau, E K O Ng, T N Leung, Y K Tong, K C A Chan, Y M D Lo
}

$\mathrm{T}$ he discovery of fetal DNA in the plasma of pregnant women $^{1}$ has led to the development of promising approaches for non-invasive prenatal diagnosis. ${ }^{2-6}$ However, as fetal and maternal DNA species co-exist in maternal plasma, these DNA based diagnostic applications depend largely on the use of genetic markers that would allow the discrimination between fetal and maternal DNA (for example, the Y chromosome of a male fetus), and thus, a particular genetic marker could generally only be used in a proportion of pregnancies. This situation has prompted a quest by many laboratories to develop fetal nucleic acid markers that are independent of sex or polymorphism.

The detection of fetal RNA in maternal plasma ${ }^{7}$ offers new possibilities for non-invasive prenatal investigation. This field has recently taken on new momentum as robust methods for plasma RNA extraction have been developed ${ }^{8}$ and circulating RNA has been shown to be surprisingly stable, ${ }^{9}$ possibly through an association with particulate matter. ${ }^{8}$ Furthermore, recent studies have identified the placenta as a significant source of such circulating fetal RNA. ${ }^{10}$ Hence, placental expressed mRNA transcripts, such as those coding for human placental lactogen $(h P L)$, human chorionic gonadotropin $\beta$ subunit ( $\beta h C G$ ), ${ }^{10}$ and corticotropin releasing hormone $(C R H),{ }^{11}$ have been shown to be detectable in maternal plasma. Quantitative assays have been developed for the measurement of these circulating mRNA transcripts. ${ }^{8}$ The pregnancy specificity of these mRNA species has been demonstrated by their rapid clearance from maternal plasma after delivery. ${ }^{10}{ }^{11}$ Thus, the detection in maternal plasma of mRNA transcripts derived from the plasma offers new avenues for the development of fetal specific nucleic acid markers that are independent of sex and polymorphism for the noninvasive prenatal assessment of all pregnancies. ${ }^{12}$ The clinical value of such an approach has been demonstrated by the observation of elevated CRH mRNA concentrations in the plasma of pre-eclamptic pregnant women, when compared with normal pregnancies matched for gestational age. ${ }^{11}$

Despite the promise shown by circulating placental mRNA in maternal plasma, several important questions remain to be explored. First, it is important to demonstrate empirically that circulating placental mRNA in maternal plasma would allow non-invasive placental gene expression profiling. Second, the previous choice of $h P L, \beta h C G^{10}$ and $C R H^{11}$ was essentially the result of the "candidate transcript" selection, based on the biological characteristics of a particular transcript. To streamline the development of further plasma RNA markers, a high throughput, generic approach is required. In this study, we systematically addressed each of these two important questions. We first investigated the feasibility of an approach based on an oligonucleotide microarray (Affymetrix) for the efficient development of new placental specific mRNA markers that could be detected in maternal plasma. We further sought to provide direct

\section{Key points}

- The recent demonstration of the presence of placental specific RNA markers in maternal plasma has made possible the development of a new generation of fetal nucleic acid markers for non-invasive prenatal investigation.

- Gene expression profiles between placental tissues and corresponding peripheral blood from pregnant women in their first and third trimesters were compared by oligonucleotide micro-array analysis with the aim of developing a systematic and high throughput strategy for the identification of new fetal RNA markers in maternal plasma.

- Panels of potentially fetal specific mRNA markers in maternal plasma were identified and six transcripts were selected for further evaluation by real time quantitative reverse transcriptase polymerase chain reaction assays.

- The detectability of transcripts in maternal plasma was directly related to their respective expression levels in placental tissues and correlations were found between the relative abundance of these six transcripts in maternal plasma and that in the placental tissues.

- Maternal plasma RNA analysis may represent a new tool for the non-invasive gene expression profiling of the fetus.

empirical evidence that maternal plasma RNA analysis did indeed allow the performance of non-invasive prenatal gene expression profiling.

\section{MATERIALS AND METHODS}

The study was performed in two stages. Initially, placental tissue gene expression profiles in both the first and third trimesters of pregnancy were systematically identified using oligonucleotide micro-arrays. This was followed by the development of a number of assays based on real time quantitative reverse transcriptase polymerase chain reaction (QRT-PCR) for the detection of six of the identified placental

Abbreviations: $\mathrm{CRH}$, corticotropin releasing hormone; CVS, chorionic villus sampling; GAPDH, glyceraldehyde-3-phosphate dehydrogenase; hPL, human placental lactogen; KISS1, KiSS-1 metastasis-suppressor; $P L A C 1$, placenta-specific 1; QRT-PCR, quantitative reverse transcriptase polymerase chain reaction; TFPI2, tissue factor pathway inhibitor $2 ; \beta$ globin, $\beta$ chain of haemoglobin; $\beta$ hCG, human chorionic gonadotropin $\beta$ subunit 
expressed genes in maternal plasma. The RNA transcripts studied were assessed both for their detectability in maternal plasma, and the correlation of their levels between plasma and placental tissues. All placental and blood samples used in this study were collected with informed consent from healthy women with uncomplicated singleton pregnancies, who attended the Department of Obstetrics and Gynaecology at the Prince of Wales Hospital, Hong Kong. The study was approved by the Clinical Research Ethics Committee.

\section{Identification of placental gene expression profiles}

Five each of first trimester (gestational age range: 912 weeks) and third trimester (gestational age range: 3840 weeks) placental tissue samples were obtained from pregnant women by chorionic villus sampling (CVS) before abortions or immediately after elective caesarean delivery, respectively. The placental tissue samples were stored in RNAlater $^{\mathrm{TM}}$ (Ambion ${ }^{\circledR}$, Austin, TX) immediately upon collection and kept at $-80^{\circ} \mathrm{C}$ until RNA extraction. Six millilitres of peripheral blood were collected concurrently at the time of tissue collection and stored in PAXgene ${ }^{\mathrm{TM}}$ blood RNA tubes (PreAnalytiX, Hombrechtikon, Switzerland).

Total RNA from placental tissues was extracted with the Trizol Reagent (Invitrogen, Carlsbad, CA) and purified with an RNeasy mini-kit (Qiagen, Hilden, Germany) following the manufacturer's protocols. Total RNA from peripheral blood was extracted by the PAXgene ${ }^{\mathrm{TM}}$ blood RNA kit (PreAnalytiX) according to manufacturer's instructions, with the inclusion of DNase treatment (RNase-Free DNase Set, Qiagen).

For each sample, $10 \mu \mathrm{g}$ of the extracted RNA were labelled and hybridised to the GeneChip ${ }^{\circledR}$ Human Genome U133A Arrays (Affymetrix, Santa Clara, CA) according to the manufacturer's instructions. After hybridisation, each array was washed and stained in a GeneChip ${ }^{\circledR}$ Fluidics Station 400 (Affymetrix). The chips were scanned with the GeneArray Scanner (Affymetrix) and analysed using the GeneChip ${ }^{\circledR}$ Microarray Suite 5.0 (Affymetrix).

\section{Quantitative assessment of placental expressed RNA transcripts in maternal plasma using real time QRT- PCR}

Paired placentas and maternal whole blood samples from 10 first and 10 third trimester pregnancies were collected. Peripheral blood samples from 10 pregnant women before and after delivery were also obtained. The placental tissues were processed as described above. Twelve millilitres of the blood samples were collected into EDTA tubes and were centrifuged at $1600 \times \mathrm{g}$ for $10 \mathrm{~min}$ at $4^{\circ} \mathrm{C}$. Plasma was then carefully transferred into plain polypropylene tubes. The plasma samples were re-centrifuged at $16000 \times \mathrm{g}$ for $10 \mathrm{~min}$ at $4^{\circ} \mathrm{C}$. Supernatants were collected into fresh polypropylene tubes. RNA extraction from maternal plasma was performed as previously described. ${ }^{8}$ DNase treatment (RNase-Free DNase Set, Qiagen) was carried out to remove any contaminating DNA.

The study focused on the assessment of six placental expressed mRNA transcripts that were identified from the placental micro-array gene expression profiles, including human placental lactogen $(h P L)$, human chorionic gonadotropin beta subunit $(\beta h C G)$, corticotropin releasing hormone $(C R H)$, tissue factor pathway inhibitor 2 (TFPI2), KiSS-1 metastasis-suppressor (KISSI), and placenta-specific 1 (PLAC1). Quantitative analysis of mRNA from two transcripts that are not placental specific-glyceraldehyde-3-phosphate dehydrogenase (GAPDH) and $\beta$ chain of haemoglobin $(\beta$-globin $)$-was performed as controls.
QRT-PCR assays for the detection of GAPDH ${ }^{8}, h P L, \beta h C G,{ }^{10}$ and $C R H^{11}$ mRNA were described previously. The primer sequences for the TFPI2 assay were 5' - ACAAATTTCTACACC TGGGAGGC - 3' (sense) and 5' - CGGCAAACTTTGGGAACT TTT $-3^{\prime}$ (antisense) and the dual labelled fluorescent probe was $5^{\prime}$-(FAM) TGCGACGATGCTTGCTGGAGGA (TAMRA) $-3^{\prime}$, where FAM and TAMRA represent 6-carboxyfluorescein and 6-carboxytetramethylrhodamine, respectively. The primer sequences for KISSI quantification were 5'- GCCCA GGCCAGGACTGA -3' (sense) and 5'- GCCAAGAAACCA GTGAGTTCATC - $3^{\prime}$ (antisense), and the dual labelled fluorescent probe was 5'-(FAM) CCTCAAGGCACTTCTAGGA CCTGGCTCTTC (TAMRA)-3'. The PLACl assay primer sequences were 5' - ATTATCCCCAGCTGCCAGAA -3' (sense) and 5'- GCAGCCAATCAGATAATGAACCA -3' (antisense), and the dual-labelled fluorescent probe was 5'-(FAM) AAGAAATCCTCACTGGACGGCTTCCTG (TAMRA)-3'. The primer sequences for the $\beta$-globin assay were $5^{\prime}$ - GCTGCACTG TGACAAGCTGC - $3^{\prime}$ (sense) and 5' GCACACAGACCAGCA CGTTG -3' (antisense), and the fluorescent probe was 5'(FAM) CGTGGATCCTGAGAACTTCAGGCTC (TAMRA)-3'.

Calibration curves for hPL, $\beta h C G, C R H, T F P I 2$, KISS1, $P L A C 1$, and $\beta$-globin mRNA quantification were prepared by serial dilutions of high performance liquid chromatography purified single stranded synthetic DNA oligonucleotides ${ }^{13}$ (Proligo, Singapore) specific for the respective amplicons, with concentrations ranging from $1 \times 10^{6}$ copies to 10 copies. The sequences of the synthetic DNA oligonucleotides for $h P L$, $\beta h C G,{ }^{10}$ and $C R H^{11}$ calibration were described previously. The sequences of the synthetic DNA oligonucleotides for TFPI2, KISS1, PLAC1, and $\beta$-globin calibrators were 5' - CGCCAACA ATTTCTACACCTGGGAGGCTTGCGACGATGCTTGCTGGAGG ATAGAAAAAGTTCCCAAAGTTTGCCGGCTG -3', 5' - CTGCCC AGGCCAGGACTGAGGCAAGCCTCAAGGCACTTCTAGGACC TGGCTCTTCTCACCAAGATGAACTCACTGGTTTCTTGGCAG -3', 5' - ACAAATTATCCCCAGCTGCCAGAAGAAGAAATCCTC ACTGGACGGCTTCCTGTTTCCTGTGGTTCATTATCTGATTGG

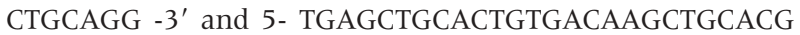
TGGATCCTGAGAACTTCAGGCTCCTGGGCAACGTGCTGGTC TGTGTGCTGG $-3^{\prime}$ respectively. Except for GAPDH mRNA, absolute concentrations of all transcripts were expressed as copies/ng of total placental RNA and copies/ml of plasma for placental tissues and maternal plasma, respectively. The calibration curve for GAPDH quantification was prepared by serial dilutions of human total RNA. ${ }^{8}$

The QRT-PCR reactions were set up according to the manufacturer's instructions (EZ rTth RNA PCR reagent set, Applied Biosystems, Foster City, CA) in a reaction volume of $50 \mu \mathrm{l}$. The QRT-PCR assays were carried out in a combined thermal cycler and fluorescence detector (ABI Prism 7700, Applied Biosystems, Foster City, CA). The reaction conditions for the GAPDH, ${ }^{8} h P L, \beta h C G,{ }^{10}$ and $C R H^{11}$ QRT-PCR assays were described previously. For the other three transcripts, the PCR primers (Geneset Oligos, Singapore) were used at concentrations of $200 \mathrm{nM}$ for TFPI2 and $\beta$-globin, $300 \mathrm{nM}$ for KISSI, and $400 \mathrm{nM}$ for PLAC1. The fluorescent probes (Applied Biosystems) were used at concentrations of $80 \mathrm{nM}$ for TFPI2, $150 \mathrm{nM}$ for KISS1, $200 \mathrm{nM}$ for PLACl, and $300 \mathrm{nM}$ for $\beta$-globin. Before performing QRT-PCR, contaminating DNA in the extracted placental tissue RNA was removed by DNase I digestion (Invitrogen, Carlsbad, CA) according to the manufacturer's recommendations. $0.4 \mathrm{ng}$ of extracted placental RNA and $6 \mu \mathrm{l}$ of extracted plasma RNA were used for amplification. Multiple negative water blanks were included in every analysis.

The thermal profiles used for TFPI2, KISS1, PLAC1, and $\beta$-globin mRNA analysis were as follows: the reaction was initiated at $50^{\circ} \mathrm{C}$ for $2 \mathrm{~min}$ for the included uracil $\mathrm{N}$-glycosylase to act, followed by reverse transcription at 
Table la Microarray detection of the 50 most highly expressed genes in CVS tissues

\begin{tabular}{|c|c|c|c|}
\hline Transcript & Probe set ID & GenBank accession no & Signals (median) \\
\hline 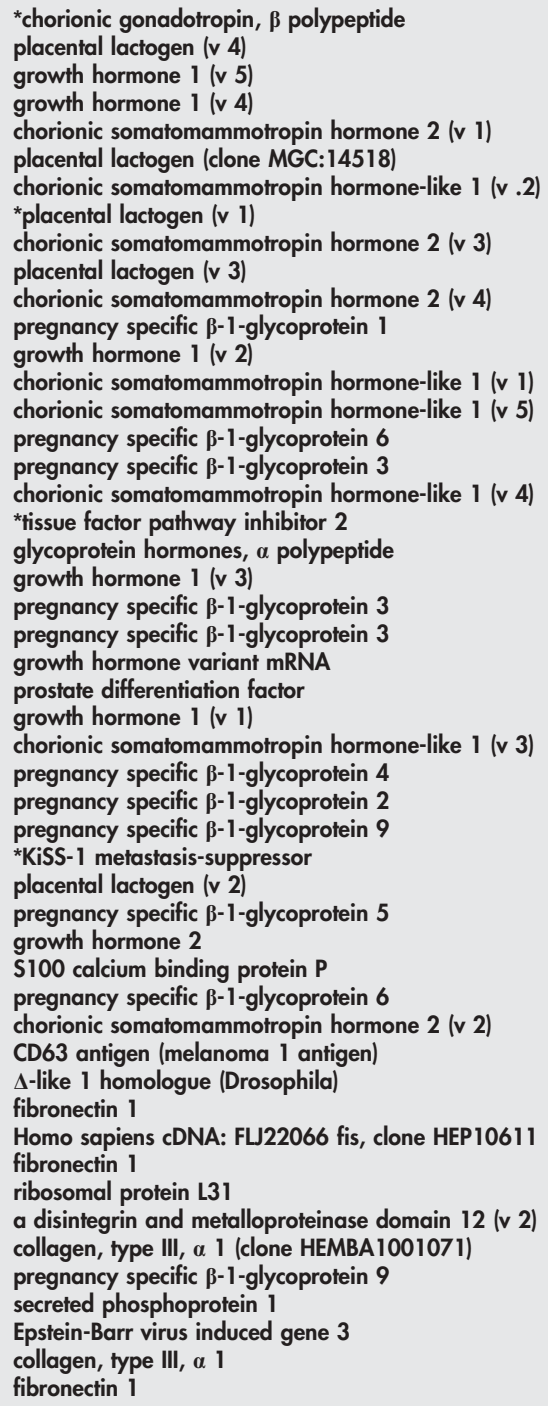 & 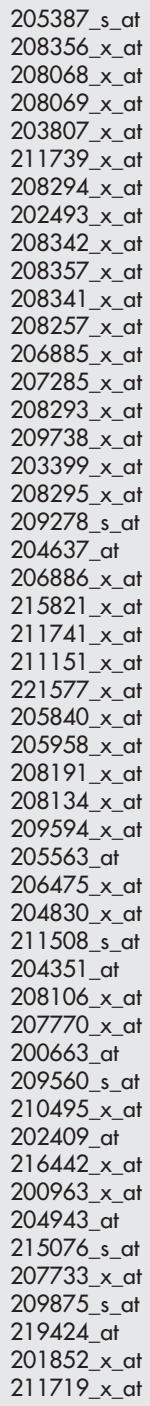 & $\begin{array}{l}\text { NM_000737.1 } \\
\text { NM_022642.1 } \\
\text { NM_022562.1 } \\
\text { NM_022561.1 } \\
\text { NM_020991.2 } \\
\text { BC005921.1 } \\
\text { NM_022578.1 } \\
\text { NM_001317.2 } \\
\text { NM_022645.1 } \\
\text { NM_022641.1 } \\
\text { NM_022646.1 } \\
\text { NM_006905.1 } \\
\text { NM_022559.1 } \\
\text { NM_001318.2 } \\
\text { NM_022581.1 } \\
\text { M31125.1 } \\
\text { NM_021016.1 } \\
\text { NM_022580.1 } \\
\text { L27624.1 } \\
\text { NM_000735.2 } \\
\text { NM_022560.1 } \\
\text { ABO19570.1 } \\
\text { BC005924.1 } \\
\text { AF185611.1 } \\
\text { AF003934.1 } \\
\text { NM_000515.2 } \\
\text { NM_022579.1 } \\
\text { NM_002780.1 } \\
\text { NM_031246.1 } \\
\text { M34421.1 } \\
\text { NM_002256.1 } \\
\text { NM_022640.1 } \\
\text { NM_002781.1 } \\
\text { AF006060.1 } \\
\text { NM_005980.1 } \\
\text { NM_002782.3 } \\
\text { NM_022644.1 } \\
\text { NM_001780.1 } \\
\text { U15979.1 } \\
\text { AF130095.1 } \\
\text { X07868 } \\
\text { AK026737.1 } \\
\text { NM_000993.1 } \\
\text { NM_021641.1 } \\
\text { AU144167 } \\
\text { NM_002784.1 } \\
\text { M83248.1 } \\
\text { NM_005755.1 } \\
\text { Al813758 } \\
\text { BC005858.1 }\end{array}$ & 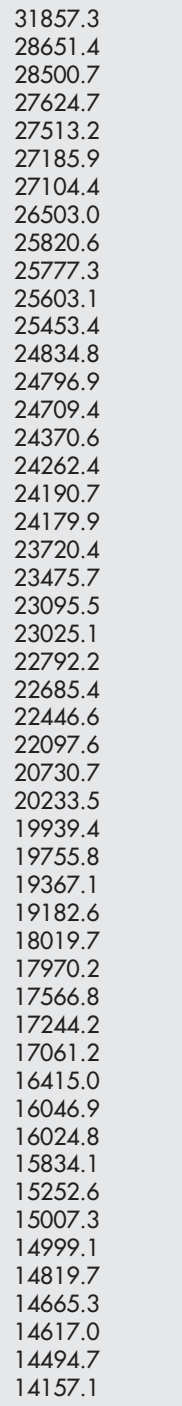 \\
\hline
\end{tabular}

$60^{\circ} \mathrm{C}$ for $30 \mathrm{~min}$. After a $5 \mathrm{~min}$ denaturation at $95^{\circ} \mathrm{C}, 40$ cycles of PCR were carried out using denaturation at $92^{\circ} \mathrm{C}$ for $15 \mathrm{~s}$ and $1 \mathrm{~min}$ annealing and extension at $56^{\circ} \mathrm{C}$ for $P L A C 1,57^{\circ} \mathrm{C}$ for TFPI2 and KISS1, and $58^{\circ} \mathrm{C}$ for $\beta$-globin.

Statistical analyses were performed using the Sigma Stat 2.03 software (SPSS).

\section{RESULTS}

Identification of placental specific genes by high density oligonucleotide array analysis of placental tissues and paired maternal blood samples

Gene expression profiles of five first trimester chorionic villus samples and five term placentas were obtained by microarray analysis of each individual tissue sample. A total of 7226 and 8871 gene transcripts were found to be expressed in the chorionic villus samples and term placentas, respectively. We have previously reported that circulating DNA in the plasma of normal individuals is predominantly derived from haematopoietic cells. ${ }^{14}$ Thus, we hypothesise that much of the background maternal nucleic acids in maternal plasma also originate from the haematopoietic compartment. As the ultimate aim of the study was to identify placental expressed transcripts that are fetal specific amongst the circulating RNA molecules in maternal plasma, we further obtained the gene expression profiles of paired maternal whole blood and compared these profiles with those of the corresponding placental tissues using the GeneChip ${ }^{\circledR}$ Microarray Suite 5.0 software (Affymetrix). Fetal specific placental expressed transcripts in early pregnancy were identified by selecting transcripts whose expression levels were "increased" in the CVS tissues when compared to the corresponding whole blood samples in all five comparisons. Fetal specific transcripts of late pregnancy were similarly identified from the term placentas when compared with the paired maternal whole blood samples. After these procedures, transcripts that were highly expressed in both the placental tissues and maternal blood cells were eliminated, resulting in panels of 1245 and 1743 transcripts, identified for the first and third trimesters of pregnancy respectively. The transcripts on these two panels were then sorted in descending order according to the medians of the five CVS or five term placental tissue micro-array expression signals (see Tables la and b for the 
Table $1 \mathrm{~b}$ Microarray detection of the 50 most highly expressed genes in term placental tissues

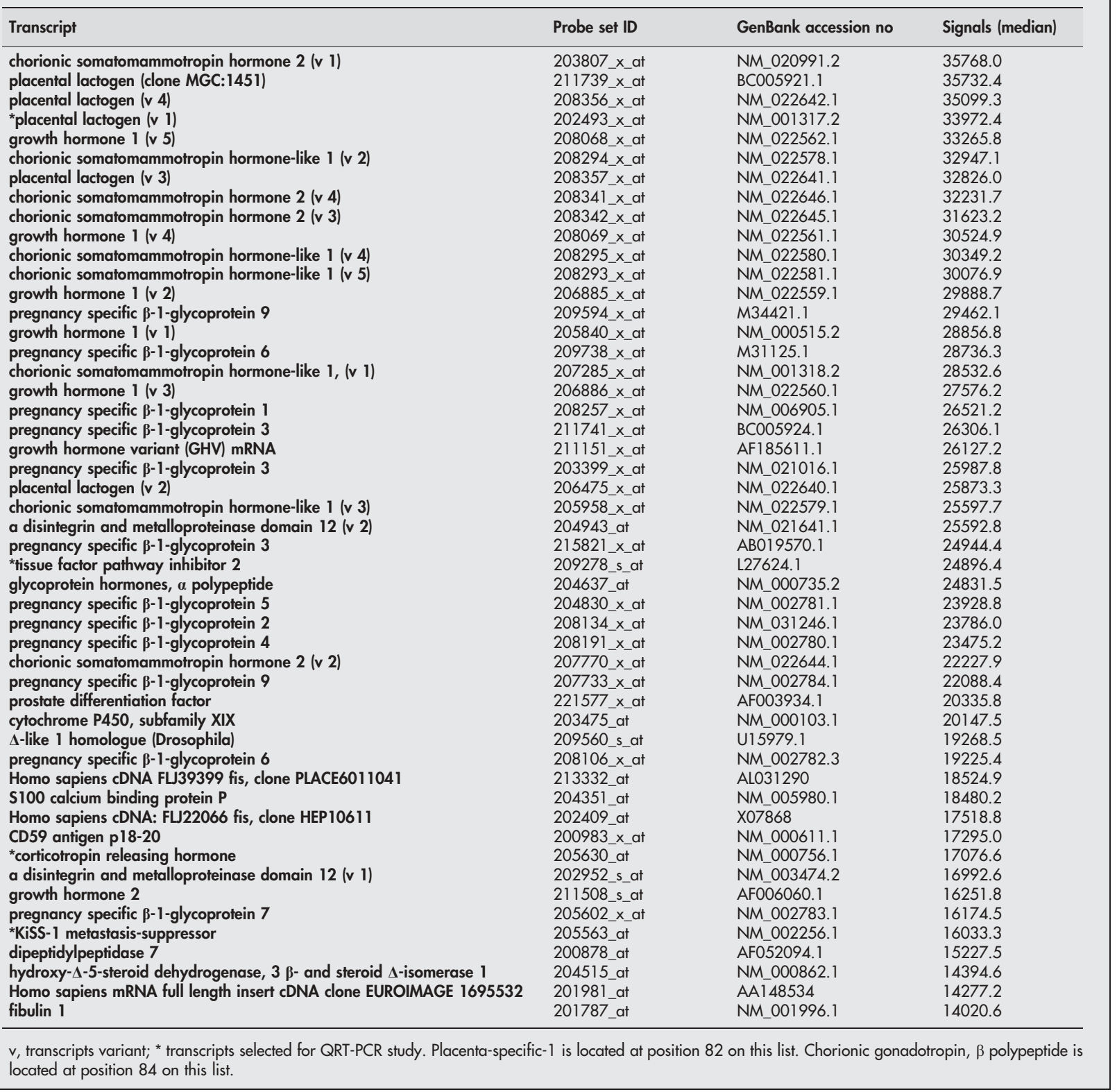

50 most highly expressed transcripts for early and late pregnancies, respectively). The two resultant panels consist of candidate transcripts that are potentially detectable in maternal plasma as fetal specific markers. The strategy used in the identification of such fetal specific markers is summarised in fig 1 . The fact that three mRNA transcripts-namely, $h P L, \quad \beta h C G,^{10}$ and $C R H^{11}$ - that we have previously demonstrated to be detectable in maternal plasma are present in the list provides independent validation of our approach. However, as our previous studies have not included information on the gene expression levels in the placenta and maternal plasma, these three transcripts were included in further analysis, together with the three novel markers identified in the list, namely, TFPI2, KISS1, and PLAC1. To compare the relative gene expression profiles between placental tissues and maternal plasma, we purposely selected transcripts that are located at different positions on the list. The median micro-array expression signal intensities of these six transcripts are summarised in Table 2 . The signal intensity of each transcript was first subjected to global scaling of the overall intensities of all arrays to a target intensity value of
500, and the medians of the scaled transcript intensities in the five chorionic villus samples and five term placental tissues were determined.

\section{Development of real time QRT-PCR assays to measure placental expressed transcripts in maternal plasma}

Six one step real time QRT-PCR assays were used. The six selected placental mRNA transcripts were quantified in paired placental tissues and plasma samples from 10 first and 10 third trimester pregnant women by QRT-PCR. The six transcripts were detectable from the placental tissues of all cases. The detectability and the median concentrations of these transcripts in both the first and the third trimester maternal plasma are summarised in table 2. These results demonstrate that a significant proportion of the selected placental expressed gene transcripts identified by micro-array analysis can indeed be detected in maternal plasma. In general, transcripts with relatively higher median micro-array signal intensities are more readily detectable in maternal plasma. On the contrary, a median maternal plasma concentration of zero copy is noted for the least abundant 


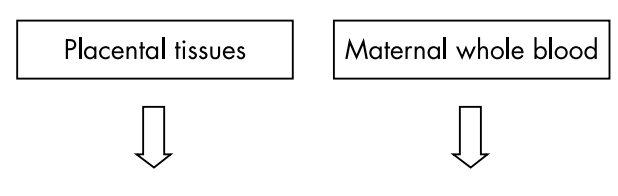

Oligonucleotide microarray

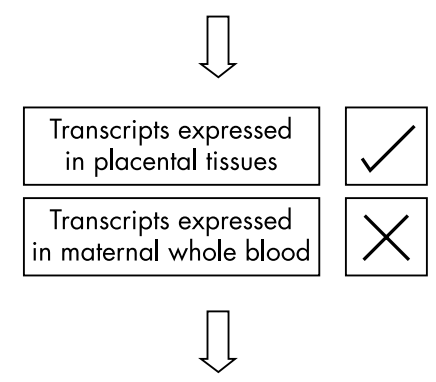

Real time quantitative RT-PCR

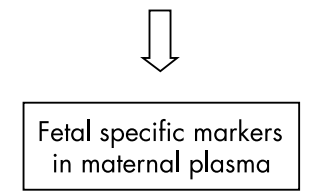

Figure 1 Outline of the strategy used for the systematic identification of pregnancy specific placental expressed mRNA markers in maternal plasma. Paired placental tissues and maternal whole blood samples are collected and subjected to oligonucleotide micro-array analysis. Transcripts with increased expression in the placental tissues relative to whole blood are selected and their detectability in maternal plasma and pregnancy specificity are evaluated by QRT-PCR on maternal plasma.

transcripts among the six studied placental expressed genes, namely, PLACl in the first trimester, and $\beta h C G$ and PLACl in the third trimester. Hence, these data suggest that placental expressed transcripts can be robustly detected in maternal plasma provided that the expression level exceeds a threshold micro-array signal.

\section{Clearance of the placental expressed transcripts from maternal plasma after delivery}

If the studied transcripts were pregnancy specific, then they would be cleared from maternal plasma after delivery. As shown in the previous studies, $h P L^{10}$ and $C R H^{11}$ mRNA molecules were cleared rapidly from maternal plasma after delivery. To investigate the clearance of TFPI2, KISS1, and PLACI mRNA from maternal plasma, plasma samples from 10 pregnant women were obtained before and at 24 hours after delivery. In the predelivery plasma samples, the median TFPI2 and KISSI mRNA concentrations were 112 copies $/ \mathrm{ml}$ and 88 copies $/ \mathrm{ml}$, respectively. Both transcripts were not

Table 2a Summary of micro-array results in chorionic villus samples and QRT-PCR results in first trimester maternal plasma of the six selected placental transcripts

\begin{tabular}{llll}
\hline Transcript & $\begin{array}{l}\text { Median CVS } \\
\text { micro-array } \\
\text { signal }\end{array}$ & $\begin{array}{l}\text { Detectability in } \\
\text { first trimester } \\
\text { plasma, } \%\end{array}$ & $\begin{array}{l}\text { Median concentration } \\
\text { in first trimester } \\
\text { plasma, copies/ml }\end{array}$ \\
\hline BhCG & 31857 & 90 & 4969 \\
hPL & 26503 & 90 & 769 \\
TFPI2 & 24180 & 70 & 38 \\
KISS1 & 19756 & 50 & 18 \\
CRH & 6941 & 70 & 16 \\
PLAC1 & 3020 & 0 & 0 \\
\hline
\end{tabular}
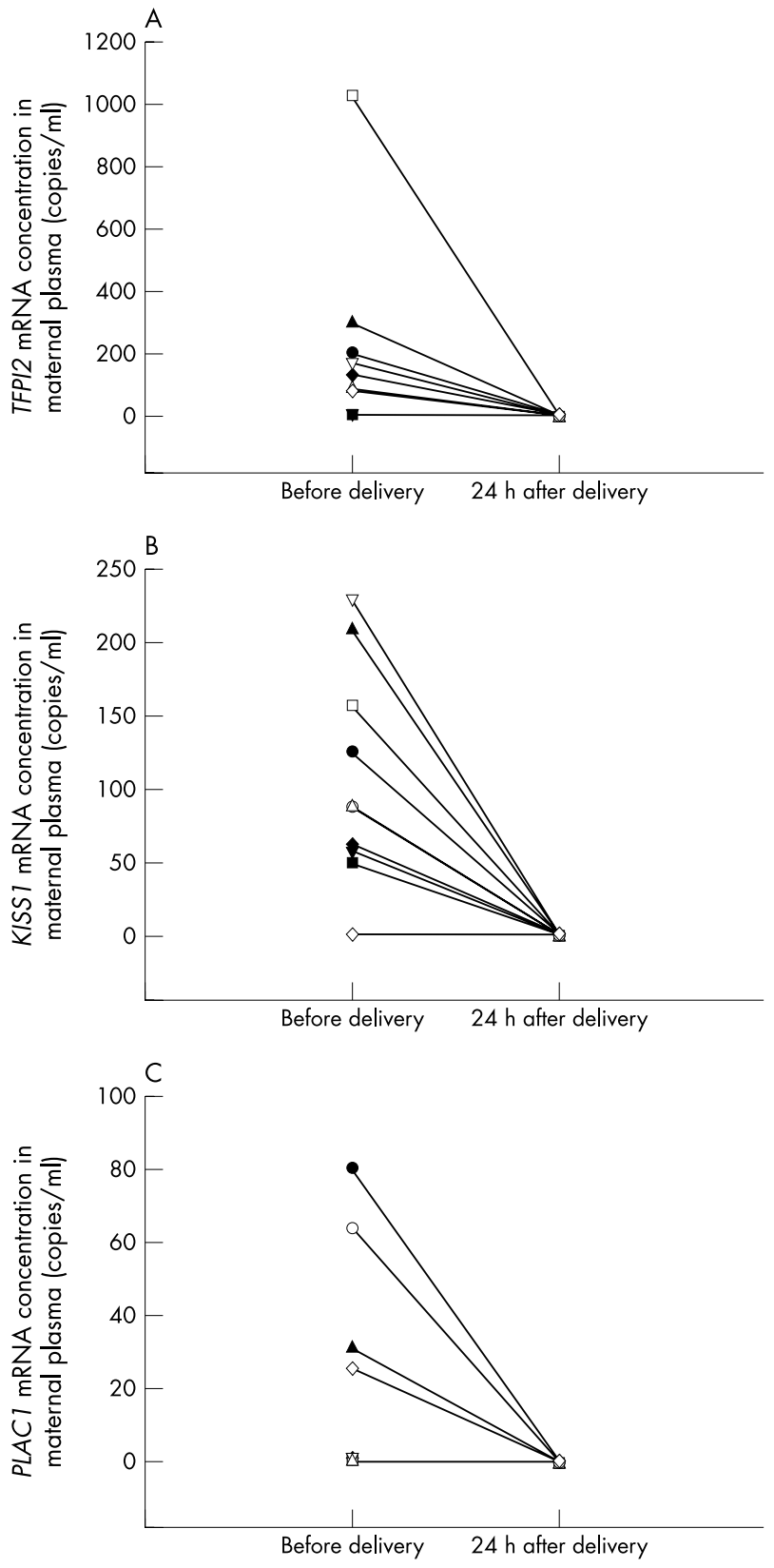

Figure 2 Clearance of placental mRNA from maternal plasma after delivery. Concentrations of (A) TFPI2 mRNA, (B) KISS1 mRNA, and (C) $P L A C 1$ mRNA in maternal plasma before delivery and at 24 hours after delivery were measured by QRT-PCR. Each line represents one plasma sample obtained from one subject.

Table $2 b$ Summary of micro-array results in term placental tissues and QRT-PCR results in third trimester maternal plasma of the six selected placental transcripts

\begin{tabular}{llll}
\hline Transcript & $\begin{array}{l}\text { Median term } \\
\text { placenta micro- } \\
\text { array signal }\end{array}$ & $\begin{array}{l}\text { Detectability in } \\
\text { third trimester } \\
\text { plasma, } \%\end{array}$ & $\begin{array}{l}\text { Median concentration } \\
\text { in third trimester } \\
\text { plasma, copies/ml }\end{array}$ \\
\hline hPL & 33972 & 100 & 14707 \\
TFPI2 & 24896 & 100 & 189 \\
CRH & 17077 & 70 & 98 \\
KISS1 & 16033 & 100 & 50 \\
BhCG & 9759 & 0 & 0 \\
PLAC1 & 9755 & 40 & 0 \\
\hline
\end{tabular}


A

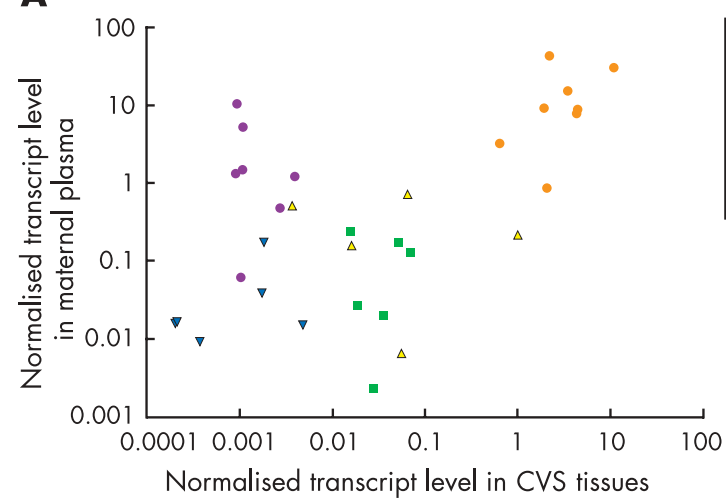

B
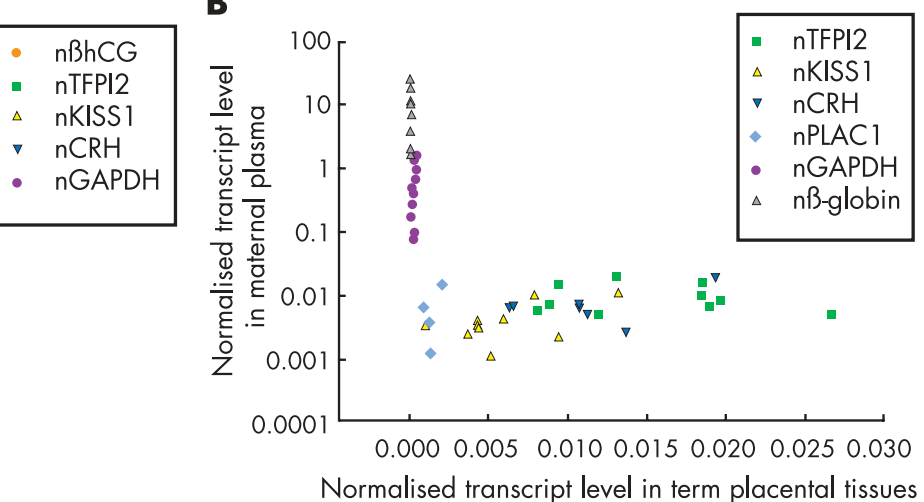

Figure 3 Correlation of normalised placental mRNA levels in maternal plasma and placental tissues. (A) Correlation of normalised placental mRNA levels between first trimester plasma and CVS. (B) Correlation of normalised placental mRNA levels between third trimester plasma and term placentas. The normalised levels of $\beta$ hCG (n $\beta$ hCG), TFPI2 (nTFPI2), KISS1 (nKISS1), CRH (nCRH), and PLAC1 (nPLAC1) mRNA in plasma and placental tissues were determined by QRT-PCR and indicated by the respective symbols indicated in the legends. The normalised levels for the non-placental specific transcripts, GAPDH (nGAPDH) and $\beta$-globin (n $\beta$-globin) are also indicated.

detected in any of the postpartum plasma samples (figs $2 \mathrm{~A}$ and $2 \mathrm{~B}$ for TFPI2 and KISSI mRNA, respectively). For PLAC1 mRNA, the transcript was detected in 4 of the 10 predelivery plasma samples, while no signal was detected in any of the postpartum samples (fig 2C). As a control, GAPDH mRNA was detected in all pre- and post-delivery plasma samples, with no systematic change in the concentrations (Wilcoxon test, $\mathrm{p}=0.563)$.

\section{Validation of mRNA analysis in maternal plasma for non-invasive placental gene expression profiling}

We sought to obtain direct evidence that the measurement of placental transcript levels in maternal plasma would provide an indirect measure of gene expression levels in the placenta. Paired placental and plasma samples from 10 first and 10 third trimester pregnancies were analysed for $h P L, \beta h C G$, CRH, TFPI2, KISS1, and PLACI mRNA by QRT-PCR. We reasoned that if the levels of these transcripts in maternal plasma were indeed a reflection of their respective levels in the placenta, then a positive correlation should be seen between the relative concentrations of these transcripts in the placenta and in maternal plasma. One practical approach to express the relative levels of these transcripts would be to normalise their levels with respect to a common placental specific transcript. As $h P L$ mRNA could be detected throughout pregnancy, ${ }^{10}$ we chose $h P L$ mRNA as our reference. The normalised level for each transcript was calculated by dividing the transcript level in an individual placenta or plasma sample to the corresponding $h P L$ mRNA level in the same sample. The comparison was only made for transcripts that were detectable in maternal plasma. As PLACl and $\beta h C G$ were not detectable at all in the current series of first and third trimester maternal plasma samples, respectively, they were not included in the respective analyses. Figs $3 \mathrm{~A}$ and $3 \mathrm{~B}$ show plots of the normalised transcript levels for $\beta h C G, C R H$, TFPI2, KISS1, and PLAC1 mRNA in the placentas and the paired maternal plasma. A positive correlation was seen between the placental and maternal plasma results for both the first (Spearman correlation analysis, $r_{\mathrm{s}}=0.452, \mathrm{p}<0.05$ ) and third (Spearman correlation analysis, $r_{\mathrm{s}}=0.661, \mathrm{p}<0.05$ ) trimesters. As a control, two non-placental specific mRNA transcripts were also analysed: $\beta$-globin for the third trimester and GAPDH for both first and third trimester samples. As can be seen in figs $3 \mathrm{~A}$ and $3 \mathrm{~B}$, these non-placental specific transcripts clearly did not follow the correlative trend exhibited by the placental specific transcripts. These data suggest that the placental specific mRNA levels measured from maternal plasma may be used to assess placental gene expression.

\section{DISCUSSION}

In the present study, we have developed a strategy which allows the systematic identification of a large panel of fetal specific RNA markers in maternal plasma (fig l). The strategy was devised based on the previous finding that the placenta is an important source of circulating fetal RNA in maternal plasma $^{10}$ and the predominant haematological origin of plasma DNA in normal individuals. ${ }^{14}$ High density oligonucleotide micro-arrays were used systematically to identify a large panel of placental expressed transcripts and to select the potentially fetal specific transcripts for maternal plasma detection, with genes expressed by blood cells being discarded as potential targets (Tables la and lb). Three previously identified plasma placental specific mRNA markers-coding for $h P L, \beta h C G,^{10}$ and $C R H^{11}$-were present in our generated target list and provided independent validation of our transcript selection strategy. In addition, three mRNA markers identified in the list, namely, TFPI2, KISS1, and $P L A C 1$, previously unexplored for non-invasive prenatal monitoring, were also detectable in maternal plasma by QRT-PCR provided that their placental tissue expression level was above a certain threshold. Their rapid clearance from the maternal plasma after delivery confirms the placental specificity. These findings add further weight to the validity of our strategy used for the identification of fetal specific transcripts in maternal plasma.

It is of special interest that our data demonstrate that the relative concentrations of placental mRNA in plasma directly reflected the gene expression patterns in the placenta. These results are remarkable because besides the level of placental gene expression, there may be other factors that would affect the transcript levels in maternal plasma. These factors include: (a) placental size; $(b)$ the rate of liberation of mRNA transcripts from the placenta into plasma; and $(c)$ the clearance rate of the transcripts from the plasma. $h P L$ mRNA has previously been shown to be placental specific and is expressed strongly throughout the pregnancy. ${ }^{10}$ Assuming $h P L$ mRNA release into maternal plasma is affected by the placental size variation in a similar fashion to the other studied placental transcripts, the normalisation to $h P L$ mRNA could control for placental size differences and thus we could directly compare the normalised transcript concentrations in 
plasma with the normalised gene expression levels in the placenta among different individuals. After the normalisation, it is notable that the relative rankings of the five normalised transcript levels in maternal plasma correlate well with the rankings of their expression levels in the placenta in both early and late pregnancies. This evidence demonstrates that placental gene expression indeed plays a significant and predominant role in determining the placental specific mRNA concentrations and profile in maternal plasma. The data also suggest that there are no significant differences in the liberation and clearance of the studied transcripts when compared with $h P L$, which would otherwise alter the maternal plasma mRNA profile in relation to the placental gene expression profile. Thus, fetal mRNA detection and measurement in maternal plasma may indeed be a useful tool for non-invasive prenatal placental gene expression profiling.

In contrast to the placental specific mRNA species, no correlation was observed between the plasma and placental normalised mRNA levels of non-placental specific transcripts, namely, GAPDH and $\beta$-globin. Compared with the placental specific transcripts, relatively more GAPDH and $\beta$-globin mRNA was present in the maternal plasma, which might be explained by the contribution of such non-placental specific transcripts from maternal tissues-for example, the haematopoietic cells.

In conclusion, we have demonstrated that circulating placental mRNA in maternal plasma could be used for noninvasive prenatal gene expression profiling. We have further outlined a micro-array based approach for rapidly and systematically identifying new placental mRNA markers to be used for this purpose. This development has particular implications for the generation of new markers for the studying and monitoring of conditions known to be associated with placental pathology, such as trisomy $21^{15}$ and pre-eclampsia. ${ }^{16}$ These avenues of research are currently being pursued in our laboratory. In addition, we envision that the findings of this study may have implications beyond diseases related to pregnancy. For example, as tumour associated mRNA has been detected in the plasma or serum of cancer patients, ${ }^{17}{ }^{18}$ a similar approach may be used for rapidly generating new plasma tumour markers based on RNA.

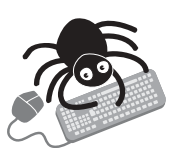

Supplemental tables are available online at http:// jimg.bmijournals.com/supplemental/

\footnotetext{
Authors' affiliations

N B Y Tsui, S S C Chim, R W K Chiu, E K O Ng, Y K Tong, K C A Chan, Y M D Lo, Department of Chemical Pathology, The Chinese University of Hong Kong, Prince of Wales Hospital, Shatin, Hong Kong SAR

T K Lau, T N Leung, Department of Obstetrics and Gynaecology, The Chinese University of Hong Kong, Prince of Wales Hospital, Shatin, Hong Kong SAR

This project is supported by the Innovation and Technology Fund (ITS/ 195/01), a Central Allocation Grant (CUHK 1/03C), and an Earmarked Research Grant (CUHK 4474/03M) from the Hong Kong Research Grants Council.
}

Conflicts of interest: The Chinese University of Hong Kong has filed patents on fetal nucleic acid analysis from maternal plasma. Aspects of fetal nucleic acid analysis in maternal plasma have been licensed to BTG and Plasmagene. YM Dennis Lo is a consultant and shareholder of Plasmagene.

Correspondence to: Y M D Lo, Department of Chemical Pathology, The Chinese University of Hong Kong, Room 38023, 1/F Clinical Sciences Building, Prince of Wales Hospital, 30-32 Ngan Shing Street, Shatin, New Territories, Hong Kong Special Administrative Region, China; loym@cuhk.edu.hk

Received 26 November 2003

Accepted for publication 1 January 2004

\section{REFERENCES}

1 Lo YMD, Corbetta N, Chamberlain PF, Rai V, Sargent IL, Redman CW, Wainscoat JS. Presence of fetal DNA in maternal plasma and serum. Lancet 1997;350:485-7.

2 Lo YMD, Hjelm NM, Fidler C, Sargent IL, Murphy MF, Chamberlain PF, Poon PM, Redman CW, Wainscoat JS. Prenatal diagnosis of fetal RhD status by molecular analysis of maternal plasma. N Engl J Med 1998;339:1734-8

3 Lo YMD, Lau TK, Zhang J, Leung TN, Chang AM, Hjelm NM, Elmes RS, Bianchi DW. Increased fetal DNA concentrations in the plasma of pregnant women carrying fetuses with trisomy 21. Clin Chem 1999;45:1747-51.

4 Lo YMD, Leung TN, Tein MS, Sargent IL, Zhang J, Lau TK, Haines CJ, Redman CW. Quantitative abnormalities of fetal DNA in maternal serum in preeclampsia. Clin Chem 1999:45:184-8.

5 Chiu RWK, Lau TK, Leung TN, Chow KCK, Chui DH, Lo YMD. Prenatal exclusion of $\beta$ thalassaemia major by examination of maternal plasma. Lancet 2002;360:998-1000.

6 Lee T, LeShane ES, Messerlian GM, Canick JA, Farina A, Heber WW Bianchi DW. Down syndrome and cell-free fetal DNA in archived maternal serum. Am J Obstet Gynecol 2002;187:1217-21.

7 Poon LLM, Leung TN, Lau TK, Lo YMD. Presence of fetal RNA in maternal plasma. Clin Chem 2000;46:1832-4.

8 Ng EKO, Tsui NBY, Lam NY, Chiu RWK, Yu SC, Wong SC, Lo ES, Rainer TH, Johnson PJ, Lo YMD. Presence of filterable and nonfilterable mRNA in the plasma of cancer patients and healthy individuals. Clin Chem 2002;48:1212-7.

9 Tsui NBY, Ng EKO, Lo YMD. Stability of endogenous and added RNA in blood specimens, serum, and plasma. Clin Chem 2002;48:1647-53.

$10 \mathrm{Ng}$ EKO, Tsui NBY, Lau TK, Leung TN, Chiu RWK, Panesar NS, Lit LC, Chan KW, Lo YMD. mRNA of placental origin is readily detectable in maternal plasma. Proc Natl Acad Sci U S A 2003;100:4748-53.

$11 \mathrm{Ng}$ EKO, Leung TN, Tsui NBY, Lau TK, Panesar NS, Chiu RWK, Lo YMD. The concentration of circulating corticotropin-releasing hormone mRNA in maternal plasma is increased in preeclampsia. Clin Chem 2003:49:727-31.

12 Ferguson-Smith MA. Placental mRNA in maternal plasma: prospects for fetal screening. Proc Natl Acad Sci U S A 2003;100:4360-2.

13 Bustin SA. Absolute quantification of mRNA using real-time reverse transcription polymerase chain reaction assays. J Mol Endocrinol 2000;25: 169-93

14 Lui YYN, Chik KW, Chiu RWK, Ho CY, Lam CW, Lo YMD. Predominant hematopoietic origin of cell-free DNA in plasma and serum after sexmismatched bone marrow transplantation. Clin Chem 2002;48:421-7.

15 Gross SJ, Ferreira JC, Morrow B, Dar P, Funke B, Khabele D, Merkatz I. Gene expression profile of trisomy 21 placentas: a potential approach for designing noninvasive techniques of prenatal diagnosis. Am J Obstet Gynecol 2002; 187:457-62.

16 Reimer T, Koczan D, Gerber B, Richter D, Thiesen HJ, Friese K. Microarray analysis of differentially expressed genes in placental tissue of pre-eclampsia: up-regulation of obesity-related genes. Mol Hum Reprod 2002;8:674-80.

17 Lo KW, Lo YMD, Leung SF, Tsang YS, Chan LYS, Johnson PJ, Hjelm NM, Lee JC, Huang DP. Analysis of cell-free Epstein-Barr virus associated RNA in the plasma of patients with nasopharyngeal carcinoma. Clin Chem 1999;45:1292-4.

18 Silva JM, Rodriguez R, Garcia JM, Munoz C, Silva J, Dominguez G, Provencio M, Espana P, Bonilla F. Detection of epithelial tumour RNA in the plasma of colon cancer patients is associated with advanced stages and circulating tumour cells. Gut 2002;50:530-4. 